\section{Rheumatoide Arthritis: Synovitis-Sonografie sagt Gelenkdestruktion voraus}

Möller B et al. Synovitis in rheumatoid arthritis detected by grey scale ultrasound predicts the development of erosions over the next three years. Rheumatology (Oxford) 2020; 59: 1556-1565. doi:10.1093/rheumatology/kez460

Trotz medikamentös scheinbar gut kontrollierter Krankheitsaktivität erleiden viele Patienten mit einer rheumatoiden Arthritis (RA) strukturelle Gelenkschäden. Eine aktive Synovitis prädisponiert offenbar für ein Voranschreiten der Erosionen. Welche diagnostischen Verfahren diese prognostisch ungünstige Synovitis am besten abbildet, wird kontrovers diskutiert. Schweizerische Forscher gingen dieser Fragestellung im Rahmen einer Kohortenstudie nach.

Sie untersuchten, welche Ultraschalltechnik zur Synovitis-Detektion - der Grey-Scale-Ultraschall, der Powerdoppler bzw. die Kombination beider Verfahren - das Voranschreiten der radiologisch objektivierten Gelenkdestruktionen am besten vorhersagt. Mithilfe des schweizerischen RA-Registers identifizierten die Wissenschaftler RA-Patienten, welche zusätzlich zu einer UItraschalluntersuchung der Hand eine Basisröntgendiagnostik absolviert und im weiteren Verlauf - frühestens 6 Monate später - eine weitere Röntgenaufnahme erhalten hatten. Als primären Studienendpunkt definierten die Forscher die radiologische Veränderung des Gelenkschadens zwischen der Basis- und der Verlaufsuntersuchung. Anschließend prüften sie, wie gut die mittels Grey-Scale-Ultraschall, Powerdoppler bzw. der Kombination beider Verfahren objektivierte Synovitisausprägung in beiden
Händen - diese erfassten sie mithilfe des Swiss Sonography in Arthritis and Rheumatism (SONAR)-Score (maximaler Punktwert: 54) - den radiologischen Progress vorhersagte. Bei ihren Berechnungen berücksichtigten die Wissenschaftler eine Reihe potenzieller Störvariablen wie die initiale Krankheitsaktivität, die Erkrankungsdauer, die serologischen Befunde sowie die Behandlung mit biologischen DMARDs.

\section{Ergebnisse}

In die Analyse zum Gray-Scale-Ultraschall gingen 287 Patienten ein, in die Analyse zum Powerdoppler 259 und in die Analyse zur kombinierten Anwendung 250. Nach einer medianen Nachbeobachtungszeit von 35 Monaten stellten die Forscher bei 26, 28 und $28 \%$ der Patienten dieser 3 Gruppen ein Fortschreiten der Gelenkdestruktionen fest. Ein Powerdoppler-Score $\geq 1 / 54$, ein GrayScale-Ultraschallscore $\geq 9 / 54$ bzw. 14/54 (50. bzw. 75. Perzentile) sowie ein kombinierter Score $\geq 10$ bzw. 15/54 (50. bzw. 75 . Perzentile) waren sowohl in der Roh-als auch in der adjustierten Analyse mit einem signifikant erhöhten Risiko für einen radiologischen Progress assoziiert. In der Subgruppe der mit Biologika behandelten Patienten zeigte sich: Ein Gray-Scale-Ultraschallscore $\geq 14 / 54$ und ein kombinierter Score $\geq 15 / 54$ standen in signifikantem Zusammenhang mit einem Voranschreiten der Gelenkdestruktion. Die klinische Krankheitsaktivität hatte in diesem Kollektiv hingegen keine wesentliche diagnostische Power.

\section{FAZIT}

Die große, multizentrische Real-LifeStudie zeigt, so das Fazit der Autoren: Bei RA-Patienten stellt die mittels Gray-Scale-Ultraschall objektivierte Synovitisschwere den stärksten Prädiktor bezüglich der zukünftigen Entwicklung von Gelenkerosionen dar. Dies gilt offenbar auch für die Subgruppe der mit biologischen DMARDs behandelten Patienten. Sie hoffen zukünftig auf weitere technische und methodische Ultraschallfortschritte im Hinblick auf die Synovitis-Bildgebung.

Dr. med. Judith Lorenz, Künzell 